\title{
Calidad de vida y actividad física en personas de la tercera edad con apoyo de la Estrategia de Salud Familiar en Itajaí, SC, Brasil
}

\author{
Quality of life and physical activity of elderly people assisted by a Family \\ Health Strategy team in Itajaí, SC, Brazil
}

Tatiana Mezadri', Luciane Peter Grillo’', Leo Lynce Valle de Lacerda', Franciele Caleffi’, Márcia Reis Felipe

\begin{abstract}
Resumen
Este estudio objetiva evaluar la calidad de vida y el nivel de actividad física en las personas de la tercera edad que pertenecen a un equipo de Estrategia de Salud Familiar en Itajaí, SC. La evaluación de la calidad de vida fue realizada por medio de instrumentos genéricos elaborados por investigadores de la Organización Mundial de la Salud denominados WHOQOL-bref y WHOQOL-old. Los datos recolectados fueron analizados conforme a modelos estadísticos válidos para estos instrumentos. Para medir el nivel de actividad física se utilizó un cuestionario internacional de actividad física (IPAQ) y para el análisis de esta variable se utilizó estadística descriptiva y analítica. En relación a la calidad de vida, los resultados presentan un valor semejante en ambos instrumentos: 67,42 $\pm 17,2$ en WHOQOL-bref y $68,89 \pm 11,41$ en WHOQOL-old. Las mayores puntuaciones en la calificación de la calidad de vida de esta populación fueron para "medio-ambiente" y "muerte y morir". En cuanto al nivel de actividad física, predominaron las personas mayores activas (70\%). Al comparar la relación entre calidad de vida y la práctica de actividad física, solo se encontró asociación entre el dominio físico y el nivel de actividad física. Así, la calidad de vida es un indicador de salud y los resultados a partir de instrumentos utilizados pueden sugerir intervenciones para mejor la calidad de vida en las personas mayores.
\end{abstract}

\section{Palabras-cLave}

Persona mayor; Calidad de vida; Actividad motora

\begin{abstract}
This study aims to evaluate the quality of life and physical activity level of elderly people assisted by a Family Health Strategy team in the city of Itajai, SC, Brazil. The assessment of quality of life was carried out using generic tools developed by researchers from the World Health Organization, called WHOQOL and WHOQOL -old and the data were analyzed according to statistical models already validated for these instruments. International physical activity questionnaire (IPAQ) was used to measure the level of physical activity. Descriptive and analytical statistics were used for data analysis. Quality of life scores showed similar values in both

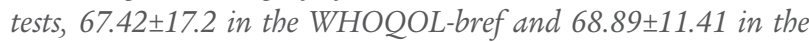
WHOQOL-old. The highest scores that assessed the quality of life of the elderly were "environment" and "death and dying". Regarding the level of physical activity, most of the elderly were active (70\%). By comparing the relationship between quality of life and physical activity, the only association found was between the physical domain and the level of physical activity. Thus, the quality of life is a bealth indicator and results from instruments used in this study can suggest intervention to improve the quality of life of the elderly population.
\end{abstract}

\section{Keywords}

Elderly; Quality of Life; Motor Activity

\section{Introducción}

Una de las mayores conquistas del siglo XX fue el aumento de la esperanza de vida como consecuencia de la modernización y del progreso científico, elevando la cantidad de personas de edad mayor en la pobla-

1 Universidad do Vale do Itajaí. Itajaí (SC)/Brasil ción mundial. Al mismo tiempo se presentó también una reducción de fertilidad, con una disminución en el número de jóvenes. Esa evolución en la configuración de la población, conocida como transición demográfica, ocurre de forma gradual en países desarrollados, posibilitando los avances sociales y políticos que se reflejan en las condiciones de vida de las personas de la tercera edad. En cuanto a los países en 
vías de desarrollo, esa transición se da de manera rápida, con cambios demográficos más profundos ${ }^{1}$.

El crecimiento de la población de personas mayores es un acontecimiento mundial y está ocurriendo en un nivel sin precedentes. En 1950, había cerca de 204 millones de personas de la tercera edad en el mundo. En 1998, menos de cinco décadas después, ése contingente alcanzó 579 millones de personas; un aumento de casi ocho millones de ancianos por año ${ }^{2}$. En Brasil, se espera que el aumento porcentual del grupo de personas mayores de 60 años sea de 13,8\% en 2020 y del $33,7 \%$ en 2060; es decir, un incremento del 20 por ciento en 40 años. En Brasil, se espera que el aumento porcentual del grupo de personas mayores de 60 años sea de $13,8 \%$ en 2020 y del 33,7\% en 2060; es decir, un incremento del 20 por ciento en 40 años. En 2030 el grupo de personas mayores de 60 años o más superará en número al grupo de niños de hasta 14 años y en 2055 al grupo de niños y jóvenes de hasta 29 años $^{3}$. Este fenómeno despierta la atención de los investigadores, ya que en Brasil existe también un interés creciente por las cuestiones ligadas a la calidad de vida de este sector de la población ${ }^{4}$.

La Organización Mundial de la Salud (OMS), define la calidad de vida como "La percepción del individuo de su lugar en el contexto de cultura y sistemas de dominios en los cuales vive, y en relación a sus objetivos, expectativas, conducta y preocupaciones" ${ }^{\prime 5}$. En el proceso de envejecimiento factores como genética, enfermedad y sedentarismo, afectan la calidad de vida de las personas mayores, por lo que la práctica de la actividad física es uno de los factores que más contribuyen para mejorar la autonomía, retardando las alteraciones fisiológicas y actuando en la prevención de las enfermedades cuando se asocia a otro tipo de atención ${ }^{6}$.

En Brasil, el sedentarismo presenta una alta prevalencia, elevando los costos, tanto directos como indirectos, para el sistema de Salud ${ }^{7}$. Con el fin de establecer una estrategia para cambiar el modelo de atención de la salud, en 2012 fueron reformuladas algunas directrices de la Estrategia de Salud en la Familia establecida en 1994 por la Política Nacional de Atención Básicsa, para la reorganización del modelo de Atención Básica en el país. El equipo de Estrategia y Salud de la Familia tienen como foco de sus acciones la familia y su comunidad ${ }^{8,9}$.

En este contexto, el objetivo de este estudio fue evaluar la calidad de vida y el nivel de actividad física de las personas de edad mayor, pertenecientes a un Equipo de Estrategias de Salud para la Familia, en el municipio de Itajaí, SC. Los estudios en esta área son de gran importancia científica y social, porque permiten la implementación de alternativas válidas de intervención de las estrategias de salud, tanto en programas geronto-geriátricos como en políticas sociales generales, con el objetivo de promover la salud en la población de la tercera edad Brasileña.

\section{Métodos}

El estudio fue una investigación transversal, de tipo exploratorio y de naturaleza cuantitativa.

La población del estudio fue compuesta por individuos con edad igual o superior a sesenta años pertenecientes a un Equipo de la Estrategia de Salud Familiar, del municipio de Itajaí, SC. La colecta de datos tuvo lugar de agosto/2009 a junio/2010.

De acuerdo con los datos del Sistema de Información de Atención Básica (SIAB), este equipo atiende a 448 ancianos distribuidos en seis micro áreas. Para el desarrollo de la investigación se seleccionaron tres micro áreas, y los individuos evaluados fueron elegidos de acuerdo a los siguientes criterios: pertenecer al Equi- 
po de Estrategias para la Salud de la Familia, tener edad igual o superior a los sesenta años, tener condiciones para responder al instrumento sin ayuda de otra persona, y firmar el formulario de consentimiento informad. Las micro áreas fueron seleccionadas en función a características de homogeneidad en cuanto a bajo nivel socioeconómico, ubicación próxima de la residencia a la unidad de salud, con tratamiento del sistema de agua y aguas residuales.

La colecta de datos fue realizada en el domicilio de las personas de edad avanzada (60 años o más) y el acceso a estas familias fue facilitado por los agentes comunitarios de la salud, responsables por la cobertura de esta área.

\section{Evaluación de los datos sociodemográficos, calidad de vida y nivel de actividad física}

Para la caracterización de la población, las personas de edad avanzada fueron entrevistadas por medio de una entrevista. Se aplicó un cuestionario socioeconómico (relación al sexo, edad, estado civil), sobre la auto percepción del estado de salud (buena o muy buena, ni mala ni buena y muy mala o débil) y grado de escolaridad.

La calidad de vida fue evaluada por medio del WHOQOL-old que permite la evaluación del impacto de la prestación de servicios y de las diferentes estructuras de asistencia social y de salud en la calidad de vida de las personas mayores, especialmente con identificación de las posibles consecuencias para las actitudes, políticas y planes en torno a un envejecimiento activo y saludable. El instrumento contiene 24 artículos de la escala de Likert apuntando a seis facetas o constructos: funcionamiento sensorial; autonomía; actividades pasadas, presentes y futuras; participación social; la muerte y morir e intimidad. Cada una de las facetas posee cuatro preguntas, por lo cual los puntajes de las facetas pueden variar entre 4 y 20 puntos. Los puntajes de estas seis facetas se combinaron para producir un puntaje total, el cual se transformó en una valoración que va de 0 a 100, donde los puntajes altos indican una alta calidad de vida y los bajos una baja calidad de vida ${ }^{11}$.

Para evaluar la calidad de vida se utilizó en común con el cuestionario WHOQOL - OLD el instrumento WHOQOL - BREF, que es una versión reducido del cuestionario original de 100 preguntas (WHOQOL - 100 ). El WHOQOL - BREF está compuesto por 26 preguntas, dos de los cuales son de carácter general con relación a calidad de vida y 24 representantes de las facetas que forman el instrumento original. Se compone de cuatro dominios de calidad de vida y cada dominio tiene como objetivo analizar respectivamente: estado físico, estado psicológico, relaciones sociales y medio ambiente. Los escores finales de cada dominio son calculado por una sintaxis, que considera la respuesta de cada pregunta que compone el de dominio lo que resulta en las puntuaciones finales en intervalos de 4 a 20, comparable al WHOQOL- 100 , que puede ser transformado en escala de 0 a $100^{10}$.

Para medir el nivel de actividad física de la población se utilizó el Cuestionario Internacional de Actividad Física (IPAQ), versión corta, propuesto por la Organización Mundial de la Salud ${ }^{12}$ y aplicado en forma de entrevista, considerando la edad de los entrevistados. El instrumento consta de cinco preguntas sobre frecuencia, duración e intensidad (vigorosa y moderada) de la actividad física realizada en los últimos 7 días, así como el caminar y el tiempo sentado en un día laborable. Los individuos fueron clasificados como muy activos, activos, insuficientemente activos y sedentarios.

Para el análisis estadístico de los resultados, en relación a las actividades físicas asociadas a WHOQOL-bref y WHOQOL-old, las clasificaciones fueron separadas 
en dos grupos: sedentarios/insuficientemente activos y activos/muy activos.

Todas las cuestiones de los instrumentos fueron completadas. Los datos colectados para evaluar la calidad de vida por medio de los instrumentos WHOQOL-bref y WHOQOL-old fueron analizados con el programa estadístico Statistica versión 10.0. Todos estos datos fueron calculados para cada sujeto investigado. El desempeño colectivo, en términos de calidad alcanzada, fue obtenido por el agrupamiento de respuestas, conforme a las secciones ${ }^{10}$.

Para comprobar la normalidad de los datos se aplicó la prueba no paramétrica D de Kolmogorov-Smirnov para los escores de los dominios WHOQOL-brefy WHOQOL-old a un nivel de significación del 5\%. Los cuatro dominios del WHOQOL-bref se presentaron normal ( $\mathrm{p}>0,10)$. De los seis dominios WHOQOL-old, cuatro tuvieron $\mathrm{p}>0,10 \mathrm{y}$ dos (actividades pasadas, presentes $\mathrm{y}$ futuras y participación social) mostraron $p>0,05$. De esta manera se consideró la distribución normal de las puntuaciones en los diez dominios evaluados.

Para evaluar el nivel de actividad física se utilizó la estadística descriptiva: media y desviación estándar y se aplicó la prueba chi cuadrado para verificar asociación entre los niveles de actividad física según el sexo, test t para identificar diferencias en las medias entre dominios de calidad de vida y niveles de actividad física y ANOVA con test Tukey paea evaluar las diferencias entre dominios. Para todas las análisis fue adoptado un nivel de significancia de 0,05.

\section{Aspectos éticos}

Todos los evaluados fueron invitados para una actividad de promoción y educación de la salud, en la cual se realizó una plática y orientación individual que abarcó los temas de estilo de vida saludable y actividad física. Los resultados individuales fueron entregados a los agentes comunitarios de la salud de cada micro área, para que realizaran la devolución a los ancianos y cuando fue necesario, fueron remitidos a especialistas profesionales de la salud.

El proyecto fue aprobado por la Comisión de Ética en Investigación de la UNIVALI, protocolo $n^{\circ} 258 / 09$ y fue financiado por el programa de Investigación Institucional PROBIC de la Universidad do Vale de Itajaí (Edital 02/2009).

\section{Resultados}

Se evaluaron 99 ancianos, 67,7\% ( $n=67)$ del sexo femenino y 32,3\% ( $n=32)$ del sexo masculino. En las características sociodemográficas de las personas evaluadas, se observa la predominancia de individuos entre los 60 y 74 años (66,0\%), casados $(52,5 \%)$ y con hasta ocho años de escolaridad (72,7\%). En este estudio, $41,4 \%$ de las personas de edad avanzada evaluaron su salud como buena o muy buena, 36,4\% ni mala ni buena y $22,0 \%$ muy mala o débil.

La Figura 1 presenta los resultados de la calidad de vida de los ancianos de acuerdo con los dominios de WHOQOL-bref. Puede verificarse que el mayor resultado fue para el valor "físico" $(73,3 \pm 13,7)$ y el menor fue para el "medio ambiente" $(66,8 \pm 16,6)$, siendo significativas estas diferencias. Destacamos que los resultados son evaluados en una escala de 0 a 100 , en cuanto a mayor resultado, mejor la calidad de vida.

Los datos presentados en la Tabla 1 muestran el nivel de actividad física de los ancianos con relación al sexo. Se observa una predominancia de individuos activos/muy activos $(70,7 \%)$ y que no hubo asociación estadística entre sexo y nivel de actividad física $(\mathrm{p}=0,202)$. 


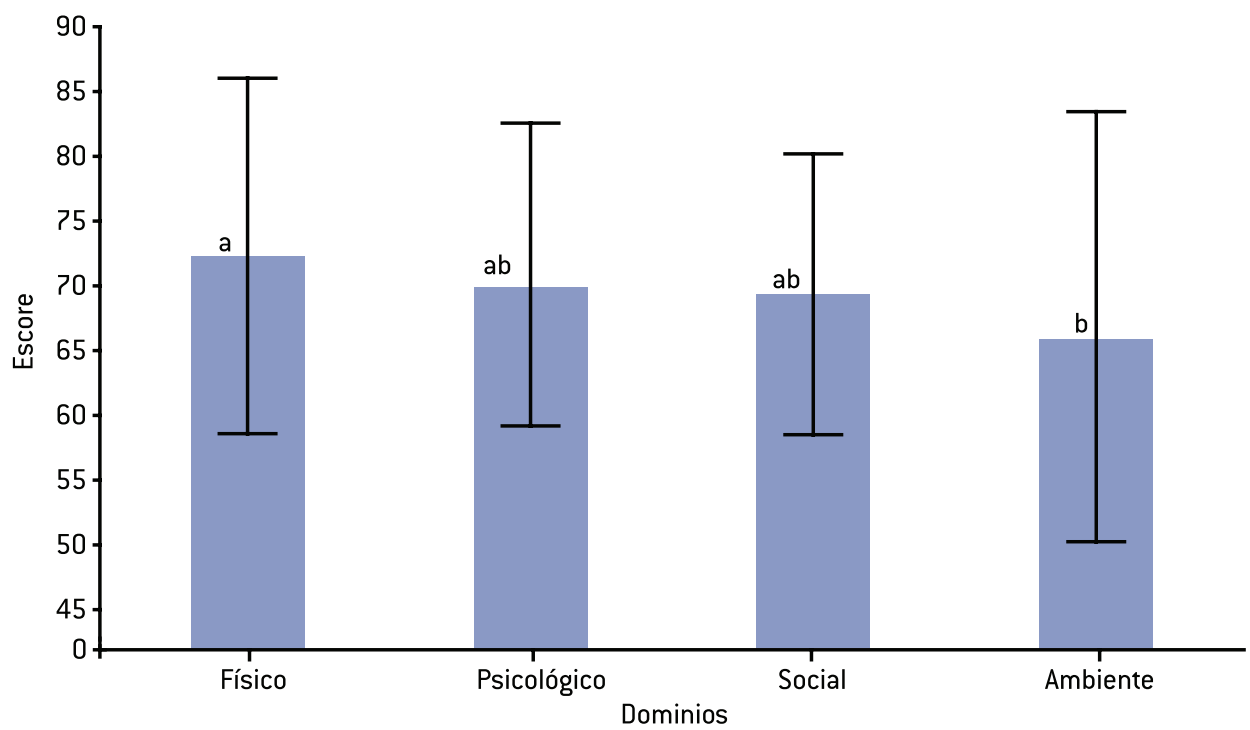

Nota: diferencias significativas $(p=0,030)$ en ANOVA. Las letras diferentes representan medias diferentes en el test Tukey.

FIGURA 1 - Calidad de vida de los ancianos de acuerdo con los dominios de WHOQOL-bref, de las personas de la tercera edad, Itajaí/SC. Se presenta la media de los dominios y la barra de desviación estándar.

Los resultados de calidad de vida de los ancianos de acuerdo a los dominios del WHOQOL-old se presentan en la Figura 2. Se observa que el resultado mayor fue para el dominio "muerte y morir" $(78,7 \pm 21,1)$ significativamente diferente de "intimidad" $(60,8 \pm 31,8)$. Los demás tuvieron valores intermediarios entre ellos.

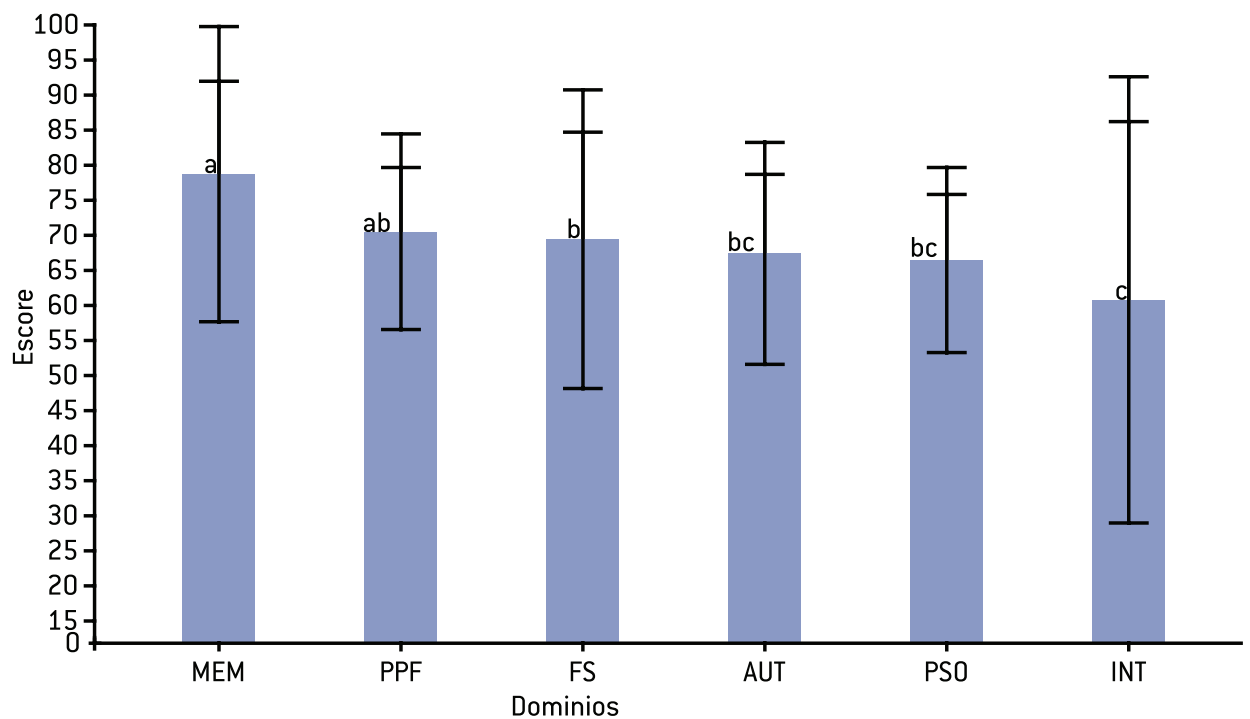

Nota: diferencias significativas $(p<0,001)$ en ANOVA. Las letras diferentes representan medias diferentes en el test Tukey. (FS= funcionamiento sensorial; AUT= autonomía; PPF= actividades pasadas, presentes y futuras; $\mathrm{PSO}=$ participación social; $\mathrm{MEM}=$ muerte y morir; INT= intimidad)

FIGURA 2 - Calidad de vida de los ancianos de acuerdo con los dominios de WHOQOL-old, de las personas de la tercera edad, Itajaí/SC. Se presenta la media de los dominios y la barra de desviación estándar. 
TABLA 1 - Clasificación del nivel de actividad física de las personas de la tercera edad con relación al sexo, Itajaí/SC.

\begin{tabular}{lccccccc} 
& \multicolumn{9}{c}{ Sexo } \\
\multirow{2}{*}{ Nivel de actividad física } & \multicolumn{2}{c}{ Masculino } & \multicolumn{2}{c}{ Femenino } & \multicolumn{2}{c}{ Total } \\
\cline { 2 - 7 } & $\mathrm{n}$ & $\%$ & $\mathrm{n}$ & $\%$ & $\mathrm{n}$ & $\%$ \\
\hline Sedentario & 6 & 18,8 & 23 & 34,3 & 29 & 29,3 \\
\hline Activo o muy activo & 26 & 81,2 & 44 & 65,7 & 70 & 70,7 \\
\hline Total & 32 & 100 & 67 & 100 & 99 & 100 \\
\hline
\end{tabular}

$\mathrm{p}=0,111$ en la prueba de chi cuadrado.

La Tabla 2 presenta una diferencia entre los cuatro dominios del WHOQOL-bref y el nivel de actividad física, con un resultado significativo solo para el dominio "físico" ( $p=0,002)$. Tampoco se encontró diferencia estadísticamente significativa entre los dominios WHOQOL-old y los niveles de actividad física, pero el dominio autonomía está muy cerca de una significación $(\mathrm{p}=0,0563)$ y el en menor grado actividades pasadas, presentes y futuras $(\mathrm{p}=0,088)$.

TABLA 2 - Escore de calidad de vida de los ancianos de acuerdo con los dominios de WHOQOL-bref, WHOQOL-old y el nivel de actividad física de las personas de la tercera edad, Itajaí/SC. Media y desviación estándar (DS).

\begin{tabular}{|c|c|c|c|c|c|}
\hline \multirow{3}{*}{ Dominio } & \multicolumn{5}{|c|}{ Nivel de actividad física } \\
\hline & \multicolumn{2}{|c|}{$\begin{array}{c}\text { Sedentario o } \\
\text { insuficientemente } \\
\text { activo }\end{array}$} & \multicolumn{2}{|c|}{ Activo o muy activo } & \multirow[t]{2}{*}{$p^{*}$} \\
\hline & Media & DS & Media & DS & \\
\hline \multicolumn{6}{|l|}{ WHOQOL-bref } \\
\hline Ambiente & 59,0 & 15,5 & 70,1 & 16,0 & 0,0021 \\
\hline Psicológico & 69,3 & 12,3 & 71,5 & 11,5 & 0,3766 \\
\hline Físico & 69,3 & 12,2 & 73,6 & 14,2 & 0,1551 \\
\hline Social & 68,4 & 8,0 & 69,7 & 11,9 & 0,5882 \\
\hline \multicolumn{6}{|l|}{ WHOQOL-old } \\
\hline Funcionamiento sensorial & 65,1 & 19,8 & 71,3 & 21,8 & 0,1914 \\
\hline Autonomía & 62,7 & 12,6 & 69,4 & 16,7 & 0,0563 \\
\hline Actividades pasadas, presentes y futuras & 66,8 & 14,9 & 72,1 & 13,4 & 0,0888 \\
\hline Participación Social & 64,0 & 12,8 & 67,5 & 13,3 & 0,2328 \\
\hline Muerte y morir & 77,8 & 19,7 & 79,1 & 21,7 & 0,7805 \\
\hline Intimidad & 60,8 & 27,8 & 60,8 & 33,5 & 0,9969 \\
\hline
\end{tabular}

*test-t de Student.

\section{Discusión}

Existen aspectos característicos y multidimensionales que definen la calidad de vida en personas de edad avanzada y están relacionados con la autoestima y con el bienestar emocional; por lo tanto, criterios de la naturaleza biológica, psicológica y sociestructural también deberían ser considerados, debido a que estos criterios son determinantes o indicadores de bienestar en la vejez ${ }^{13,14}$.

En el presente estudio hubo prevalencia de personas de la tercera edad de sexo femenino $(67,7 \%)$. La feminización en la vejez es una característica comúnmente encontrada en estudios de este tipo. Reciente estudio ${ }^{15}$ indica tasas de mortalidad masculina superiores a femenina todos los grupos de edad avanzada, ampliando la proporción de mujeres de la tercera edad.

Con relación a los datos demográficos encontrados, se verificó que la mayoría 
de los ancianos presentan una edad entre 60 y 74 años, están casados y tienen hasta el octavo grado de escolaridad. Un estudio realizado por Sprangers et al. ${ }^{16}$ mostró que factores como la edad avanzada, sexo femenino, bajo nivel de escolaridad y falta de pareja, están relacionados con un bajo nivel de calidad de vida. Otros autores complementan que la falta de actividad física, el estrés, la percepción y aceptación entorno al estado de vejez también son factores fuertemente asociados a la calidad de vida ${ }^{17}$.Aunque podemos considerar la salud como factor relevante y determinante de la calidad de vida geriátrica y la auto percepción del estado de vejez.

En un estudio realizado por Fleck et al. ${ }^{11}$ se evaluó la definición de calidad de vida y sus determinantes en personas de edad avanzada de diferentes grupos focales - ancianos saludables, enfermos (de acuerdo a la percepción subjetiva de cada individuo) y enfermos con cuidadores - y se observó una tendencia de asociación entre calidad de vida y bienestar o sentirse bien (salud, sociabilidad, soporte social, actividad física, posibilidad de dar soporte y apoyo) con el sentimiento de utilidad.

Cuando la auto percepción de salud, esta fue valorada como "buena" (42\%) por casi la mitad de los entrevistados. Gonçalves et al. ${ }^{18}$ también realizó una evaluación en cuanto a salud e identificó que gran parte de los ancianos, cerca del 66\% relataron, en respuestas libres, que la falta de salud o enfermedades les impedía "realizar actividades o trabajar", lo que se traduce en un bajo nivel de calidad de vida.

Con respecto a la actividad física, el 70,7\% de las personas de edad avanzada que participaron en el presente estudio son activos/muy activos, mientras que el $29,3 \%$ son sedentarios o insuficientemente activos. Estos resultados sorprenden si se considera que la prevalencia del sedentarismo esperada para la población brasileña, en general, es de aproximadamente $70 \%$ y las evidencias indican que ese índice aumenta con la edad ${ }^{19}$. La práctica de actividad física en esta población podría relacionarse al hecho de que Itajaí es una ciudad plana, localizada en un área litoral, lo que favorece las caminatas y el uso de bicicletas. Además; es preciso considerar que los ancianos suelen vivir en la zona central del municipio, donde es posible que la infraestructura sea mejor en cuanto a la presencia de aceras, plazas y lugares de ocio pavimentados adecuadamente.

Ramos $^{20}$ destaca que, actualmente, la capacidad funcional, surge como un nuevo paradigma de la salud para las personas mayores. Al envejecer, el individuo está sujeto a cambios que conducen a una pérdida progresiva de sus capacidades físicas o mentales, lo que causa, en algunos, una sensación de decadencia ${ }^{21}$. Con todo, la práctica de actividad física presenta beneficios en los factores relacionados con la aptitud física en las personas de edad avanzada, incluyendo la coordinación, agilidad, equilibrio y resistencia de física ${ }^{22}$. De otro lado, iniciar la actividad física después de los 60 años también puede aumentar la longevidad, mejorar la capacidad fisiológica, reducir el uso de medicamentos y ayudar en la prevención de la disminución cognitiva ${ }^{23}$.

En este estudio, de acuerdo a los datos obtenidos a partir del instrumento WHOQOL-bref, se verificó que el dominio "fisico" presenta mayor puntuación en la evaluación de Calidad de vida para las personas de la tercera edad, seguido de los dominios "psicológico", "social" y "medio ambiente". El dominio físico se refiere a el dolor y el malestar; energía y la fatiga; sueño y descanso; actividades de la vida diaria; dependencia de medicamentos o tratamientos y la capacidad trabajo ${ }^{10}$. Tavares et a ${ }^{25}$ obtuvieron resultados diferentes con el escore más alta para el dominio "social".

Los resultados referentes a los dominios del WHOQOL-old evaluaron mayor influencia del dominio "muerte y morir" asociado a la calidad de vida de esta población, seguido de "actividades pasadas, presentes y futuras", "funcionamiento 
sensorial", "autonomía”, "participación social” e "intimidad". Según Mello ${ }^{24}$ la faceta "Muerte y Morir" está relacionada con las preocupaciones, inquietudes, expectativas y temores de las personas ancianas sobre la muerte y el morir. De esta forma, el elevado valor de la puntuación sugiere que estos sentimientos no están tan presentes en los participantes.

Cuando fueron evaluados los dominios según el nivel de actividad física, se encontró una relación significativa para el dominio "ambiente" del WHOQOL-bref $(\mathrm{p}=0,002)$, o sea, las personas clasificadas como activas/muy activas tienen mayor puntaje en este dominio. El dominio "medio-ambiente" cubre los aspectos de seguridad física y protección; ambiente en el hogar; recursos financieros; disponibilidad y calidad de los cuidados de salud; oportunidad de adquirir nuevas informaciones y habilidades; participación y oportunidad de recreación y ocio; ambiente físico (contaminación/ ruido/ transito/ clima), y transporte ${ }^{10}$. Aunque los resultados no mostraron una diferencia significativa entre las facetas "autonomía" y en menor grado para "actividades pasadas, presentes y futuras" del WHOQOL-old y el nivel de actividad física, es posible que esta relación exista puesto que el valor p quedo muy próximo de 0,05 y es probable que aumentando el tamaño de la muestra pueda hallarse significancia entre estos factores. De esta forma, los resultados del presente estudio sugieren que el nivel de actividad física de las personas de la tercera edad influye en la calidad de vida, apenas en lo que respecta al dominio "ambiente" y, posiblemente, a los dominios "autonomía" y "actividades pasadas, presentes y futuras", sin influenciar en las demás facetas de la calidad de vida.

Otros estudios que evalúan la calidad de vida de las personas de tercera edad llevados a cabo por el equipo de Estrategias de Salud de la Familia mostraron que el dominio "relaciones sociales", del instrumento WHOQOL-bref, y la faceta "intimidad", del WHOQOL-old, tienen una contribución importante en la calidad de vida de los ancianos ${ }^{25}$ Los participantes de un grupo de convivencia presentaron mayor puntuación para el dominio "social" en el cuestionario WHOQOL-bref y para la faceta "funcionamiento sensorial" del WHOQOL-old. ${ }^{26}$ En Lima y col. ${ }^{27}$ se evaluó la calidad de vida de las personas registradas en el SIAB (Sistema de Información de la Atención Básica) pertenecientes a dos grupos distintos, uno de ellos formado por personas que participaban en los encuentros de la tercera edad, y otro por personas que no lo hacían. Se comprobó que, de acuerdo con la puntuación total de los dominios WHOQOL-bref y las facetas WHOQOL-old, la percepción de la calidad de vida global de las personas, de edad mayor, del grupo que participaba fue superior a la de las personas que no participaban. Así mismo, las personas del grupo que participaba presentaron una puntuación más alta para el dominio "físico" y faceta "autonomía". En este contexto se identificó que las preguntas subjetivas de la calidad de vida deben abarcar lo que ocurre con el individuo en las diferentes etapas del envejecimiento, considerando diversos criterios de la naturaleza biológica, psicológica y socio estructural ${ }^{28}$.

Este estudio tuvo limitaciones relacionadas con la dificultad que presentaron las personas de la tercera edad para concentrarse al responder el cuestionario, puesto que este era relativamente extenso y con la desconfianza que se generó por recibir personas extrañas en sus domicilios. Estas situaciones entorpecieron la colecta de datos y restringieron el número de personas evaluadas.

Así, se puede decir que en este trabajo, el perfil de la mayoría de las personas estudiadas corresponde a individuos con hasta ocho años de escolaridad, entre los 60 y 74 años de edad y que refirieran su salud como buena o muy buena. Con relación a la práctica de actividades físicas, la mayor parte de ellos tiene un nivel 
de desempeño activo o muy activo. Al evaluar los principales dominios del WHOQOL-bref y del WHOQOL-old, el estudio mostró que las mayores puntuaciones que evaluaron la calidad de vida de esta población fueron el "fisico" y "muerte y morir", respectivamente, y existe una asociación significativa entre el nivel de actividad física y el dominio "ambiente" del instrumento WHOQOL-bref.

El número de pesquisa con los ancianos en Brasil, comparando los dos instrumentos empleados en este estudio, es aún baja, lo que dificulta un debate más amplio. A partir de los resultados observados, se concluye que la calidad de vida de las personas mayores que tienen mayor nivel de actividad física es mejor que para aquellas con menor nivel de actividad física. Por lo tanto, es importante tener un plan de acciones para esta población que está creciendo, preparando la sociedad brasileña a un envejecimiento más saludable y activo.

\section{Financiamiento}

El proyecto fue financiado por el programa de Investigación Institucional (ProBIC) de la Universidad do Vale do Itajaí.

\section{Referências}

1. Carvalho JAM, Garcia RA. The aging process in the Brazilian population: a demographic approach. Cad Saúde Públ. 2003; 19(3): 725-33.

2. INSTITUTO BRASILEIRO DE GEOGRAFIA E ESTATÍSTICA (IBGE). Coordenação de Índices de Preços. Pesquisa de Orçamentos Familiares 2002-2003: primeiros resultados. Brasil e grandes regiões. Rio de Janeiro: IBGE, 2004.

3. INSTITUTO BRASILEIRO DE GEOGRAFIA E ESTATÍSTICA (IBGE). Síntese de Indicadores Sociais. Uma análise das condições de vida da população brasileira. Estudos e Pesquisas. Informação Demográfica e Socioeconômica número 29. 2013.

4. Tavares DMS, Dias FA. Capacidade funcional, morbidades e qualidade de vida de idosos. Texto contexto - enferm. 2012; 21( 1 ): 112-120.

5. Fleck MPA. Grupo WHOQOL: Desenvolvimento do WHOQOL na versão em português. [periódico na internet]. 2008 . Disponível em: http://www.ufrgs.br/psiq/whoqold.pdf.

6. Vidmar MF, Potulski AP, Sachetti A, Silveira MM, Wibelinger LM. Atividade física e qualidade de vida em idosos. Rev Saúde Pesquisa. 2011; 4(3): 417-424.

7. Veras RP. Gerenciamento de doença crônica: equívoco para o grupo etário dos idosos. Rev Saúde Pública. 2012; 46(6): 929-34.

8. Romeiro C, Nogueira JAD, Tinoco SG, Carvalho KMB. O modelo lógico como ferramenta de planejamento, implantação e avaliação do programa de Promoção da Saúde na Estratégia de Saúde da Família do Distrito Federal. Rev Bras Ativ Fís e Saúde. 2013; 18(1): 132-142.

9. Ministério da Saúde. Secretaria de Atenção à Saúde. Departamento de Atenção Básica. Política Nacional de Atenção Básica. Brasília: Ministério da Saúde; 2012.

10. Fleck MPA, Louzada S, Xavier M, Chachamovich E, Vieira G, Santos L, et al. Aplicação da versão em português do instrumento abreviado de avaliação da qualidade de vida "WHOQOL-Bref". Rev Saúde Públ. 2000; 34(2): 178-183.

11. Fleck MPA, Chachamovich E, Trentini CM. Projeto WHOQOL-OLD: métodos e resultados de grupos focais no Brasil-RS. Rev Saúde Públ. 2003; 37(6):793-99.

12. Matsudo S, Araújo T, Marsudo V, Andrade D, Andrade E, Oliveira LC, et al. Questionário internacional de atividade física (IPAQ) estudo de validade e reprodutibilidade no Brasil. Rev Bras Ativ Fís e Saúde. 2001; 6(2):5-18.

13. Vecchia RD, Ruiz T, Bocchi SCM, Corrente JE. Qualidade de vida na terceira idade: um conceito subjetivo. Rev Bras Epidemiol. 2005; 8(3): 246-52.

14. Murakami L, Scattolin F. Evaluation of functional independence and quality of life in institutionalized elderly. Rev Med Hered. 2010; 21(1):18-26

15. Ervatti LR, Borges GM, Jardim AP (Org). Mudança demográfica no Brasil no início do século XXI: subsídios para as projeções das populações. Instituto Brasileiro de Geografia e Estatística (IBGE): Rio de Janeiro, 2015. 
16. Sprangers MA, Regt EB, Andries F, Van Agt HME, Bijl RV, Boer JB, et al. With chronic conditions are associated with better or poorer quality of life? J Clin Epidemiol. 2000; 53(9): 905-07.

17. Avis NE, Assmann SF, Kravitz HM, Ganz PA, Ory M. Quality of life in diverse groups of midlife women: assessing the influence of menopause, health status and psychosocial and demographic factors. Qual Life Res. 2004; 13(5): 933-46.

18. Gonçalves LHT, Dias MM, Liz TG. Qualidade de vida de idosos independentes segundo proposta de avaliação de Flanagan. Mundo Saúde. 1999; 23(4): 214-20.

19. Mendonça TT, Ito RE, Bartholomeu T, Tinucci T, Forjaz CLM. Risco cardiovascular, aptidão física e prática de atividade física de idosos de um parque de São Paulo. Rev Bras Ciên Mov. 2004; 12(3): 57-62.

20. Ramos LR. Fatores determinantes do envelhecimento saudável em idosos residentes em centro urbano: Projeto Epidoso, São Paulo. Cad Saúde Publ. 2003; 19(3): 793-798.

21. Goldenberg J. Promoção da saúde na terceira idade: dicas para viver melhor. Atheneu: São Paulo, 2008.

22. Mazo GZ, Sacomori C, Krug RR, Cardoso FL, Benedetti TRB. Aptidão física, exerícios físicos e doenças osteoarticulares em idosos. Rev Bras Ativ Fís e Saúde. 2012; 17(4): 300-306.

23. Garcia MA, Rodrigues MG, Borega RS. O envelhecimento e a saúde. Rev Ciênc Méd: 2002; 11(3): 221-31.

24. OLIVEIRA-CAMPOS, M. et al. Impacto dos fatores de risco para doenças crônicas não transmissíveis na qualidade de vida. Ciência \& Saúde Coletiva. 2013; 18(3):873-882.

25. Tavares DMS, Gávea SA Junior, Dias FA, Santos NMF, Oliveira PB. Qualidade de vida e capacidade funcional de idosos residentes da zona rural. Rev Rene. 2011; 12(n. esp.): 895-903.

26. Serbim AK, Figueiredo AEPL. Qualidade de vida de idosos em um grupo de convivência. Rev Sci Med. 2011; 21(4): 166-172.

27. Lima LCV, Bittar CML. A percepção da qualidade de vida em idosos: um estudo exploratório. Rev Bras de Qualidade de Vida. 2012; 4(2): 1-11.

28. Santos SR, et al. Qualidade de vida do idoso na comunidade: aplicação da escala de Flanagan. Rev Latino-Am Enferm. 2002; 10(6): 757-64.

DIRECCIÓN PARA

CORRESPONDENCIA

LUCIANE PETER GRILLO

grillo@univali.br.
Universidad do Vale do Itajaí: Rua

Uruguai, 458, Bloco 6, Setor F, Itajaí-SC

CEP 88302-202.

Teléfono: (55 47) 33417932.
RECEBIDO 27/08/2015

REVISADO 14/12/2015

ACEPTADO $\quad 20 / 12 / 2015$ 